\title{
Evolução Hospitalar de Pacientes Submetidos a Assistência Circulatória com Balão Intra-Aórtico durante Intervenção Coronária Percutânea de Alto Risco: Registro InCor
}

\author{
Fábio Conejo', Luciano Nunes dos Santos', Henrique Barbosa Ribeiro', Carlos A. Campos', \\ Antônio Hélio Pozetti ${ }^{1}$, Augusto C. Lopes Jr. ${ }^{1}$, Rodrigo Barbosa Esper ${ }^{1}$, Antônio Esteves Filho', \\ André Gasparini Spadaro' ${ }^{1}$ Paulo Rogério Soares ${ }^{1}$, Marco Antonio Perin¹, \\ Expedito E. Ribeiro' ${ }^{1}$, Gilberto Marchiori ${ }^{1}$
}

\section{RESUMO}

Introdução: O balão intra-aórtico (BIA) é utilizado há décadas como dispositivo de assistência circulatória, sendo recomendado na maioria das diretrizes, apesar da fraca evidência em relação à redução da mortalidade. O objetivo deste estudo foi avaliar o uso do BIA como ferramenta adjunta na intervenção coronária percutânea (ICP). Métodos: Registro unicêntrico que analisou a evolução hospitalar de 134 pacientes consecutivos que utilizaram BIA durante ICP de alto risco ou na vigência de choque cardiogênico. Resultados: A média de idade foi de $64,7 \pm 12,5$ anos e $67,9 \%$ eram do sexo masculino. A população apresentou características de alto risco, com 33,5\% de diabéticos, $73,1 \%$ tratados na vigência de infarto do miocárdio, 68,6\% com padrão triarterial, e fração de ejeção do ventrículo esquerdo de $38,6 \pm 16,1 \%$. Fluxo TIMI $2 / 3$ foi obtido em $86,4 \%$ dos casos, sendo tratada 1,6 $\pm 0,6$ lesão/paciente. Ocorreram $18(13,4 \%)$ reinfartos, dos quais $9(6,7 \%)$ por trombose do stent, sendo $2(1,4 \%)$ pacientes encaminhados para cirurgia de revascularização miocárdica e $16(11,9 \%)$, para nova ICP. A mortalidade hospitalar foi de $61,2 \%$ e a taxa de eventos cardíacos adversos maiores (ECAM) foi de $67,2 \%$. Por análise multivariada, foram preditores de mortalidade: idade > 65 anos, ICP prévia, necessidade de diálise e padrão triarterial. Os preditores de sobrevida foram: função ventricular normal, fluxo TIMI $2 / 3$ ao final do procedimento e permanência do BIA $>72$ horas. Conclusões: Os pacientes que utilizaram BIA durante ICP apresentaram perfil clínico de risco muito alto, que se refletiu em elevada taxa de óbito. Fatores clínicos,

\author{
ABSTRACT \\ In-Hospital Outcomes of Patients Submitted to \\ Circulatory Support with Intra-Aortic Balloon \\ Pump During High Risk Percutaneous Coronary \\ Intervention: InCor Registry
}

Background: The intra-aortic balloon pump (IABP) has been used for decades as a circulatory support device, being recommended in most guidelines, despite poor evidence of mortality reduction. The purpose of this study was to evaluate the use of IABP as an adjunct therapy in percutaneous coronary intervention ( $\mathrm{PCI})$. Methods: Single center registry analyzing in-hospital outcomes of 134 consecutive patients submitted to IABP during high risk $\mathrm{PCl}$ or in the presence of cardiogenic shock. Results: Mean age was $64.7+12.5$ years and $67.9 \%$ were males. The population presented high-risk features, with $33.5 \%$ of diabetic patients, $73.1 \%$ treated in the presence of myocardial infarction, $68.6 \%$ with three-vessel disease and left ventricular ejection fraction of $38.6+16.1 \%$. TIMI $2 / 3$ flow was obtained in $86.4 \%$ of the cases and $1.6 \pm 0.6$ lesion/patient was treated. There were $18(13.4 \%)$ reinfarctions, of which $9(6.7 \%)$ were due to stent thrombosis, $2(1.4 \%)$ patients were referred for CABG and $16(11.9 \%)$ for a new PCl. Hospital mortality was $61.2 \%$ and the major adverse cardiac events (MACE) rate was $67.2 \%$. By multivariate analysis, predictors of mortality were: age > 65 years, prior $\mathrm{PCl}$, need of dialysis and three-vessel disease. Predictors of survival were: normal ventricular function, TIMI 2/3 flow at the end of the procedure and IABP utilization $>72$ hours. Conclusions: Patients

\footnotetext{
1 Instituto do Coração do Hospital das Clínicas da Faculdade de Medicina da Universidade de São Paulo (InCor/HCFMUSP) - São Paulo, SP, Brasil.

Correspondência: Expedito E. Ribeiro. Av. Dr. Enéas Carvalho de Aguiar, 44 - 3o andar - Bloco I - Hemodinâmica - Cerqueira César São Paulo, SP, Brasil - CEP 05403-900

E-mail: expribeiro@terra.com.br

Recebido em: 30/8/2011 • Aceito em: 7/11/2011
} 
angiográficos e do procedimento podem identificar variáveis que interferem independentemente na mortalidade.

DESCRITORES: Balão intra-aórtico. Choque cardiogênico. Angioplastia.

$\mathbf{N}$ as últimas décadas, a mortalidade pelas doenças cardiovasculares no Brasil vem apresentando regressão, ocasionada principalmente pelas modificações no estilo de vida e pelo desenvolvimento de diversos tratamentos farmacológicos e intervencionistas. ${ }^{1}$ Mesmo assim, as doenças cardiovasculares ainda são a principal causa de morte no País, representando mais de $40 \%$ dos óbitos, ${ }^{2}$ com destaque para o infarto agudo do miocárdio (IAM), que possui relevante impacto também no número de hospitalizações. ${ }^{1-3}$

Entre os pacientes hospitalizados que morrem em decorrência do IAM, o choque cardiogênico é a principal causa, representando, na atualidade, de $7 \%$ a $10 \%$ dos eventos isquêmicos agudos. ${ }^{4,5}$ A etiologia dessa síndrome de baixo débito é decorrente de disfunção cardíaca ocasionada por isquemia e necrose de extensa área do ventrículo esquerdo ou de complicações mecânicas, como a insuficiência mitral aguda e a ruptura de estruturas cardíacas. Apesar do tratamento intensivo introduzido nos últimos anos, como a realização da intervenção coronária percutânea (ICP) precoce e o uso da terapia farmacológica adjunta e de dispositivos de assistência circulatória, a mortalidade por choque cardiogênico ainda permanece alta. ${ }^{6-8}$

Entre os dispositivos de assistência circulatória destacam-se o balão intra-aórtico (BIA), que, desde seu desenvolvimento, a partir da década de 1960, se tornou o dispositivo de assistência circulatória mais amplamente utilizado em todo o mundo. ${ }^{9}$ Seus benefícios são atribuídos à redução da pós-carga ventricular e ao aumento da pressão de perfusão coronária, sendo utilizado para suporte hemodinâmico em pacientes com choque cardiogênico, angina refratária e ICP de alto risco. Apesar da pouca evidência a respeito da redução da mortalidade, o BIA permanece recomendado pela maioria das diretrizes ${ }^{10-12}$; contudo, ainda é pouco utilizado na prática clínica (20\% a 39\%), mesmo em países desenvolvidos. ${ }^{7,13}$

Existem poucas publicações nacionais sobre a utilização rotineira do BIA em pacientes com choque cardiogênico de etiologia isquêmica, bem como de seu benefício em pacientes submetidos a ICP de alto risco. Nesse sentido, o objetivo deste estudo foi avaliar a evolução hospitalar e os preditores de mortalidade dos pacientes submetidos a implante rotineiro de BIA como ferramenta adjunta nas ICPs. undergoing $\mathrm{PCl}$ with IABP support have a very high risk clinical profile, which led to high mortality rates. Clinical, angiographic and procedure-related factors can identify independent variables for mortality.

KEY-WORDS: Intra-aortic balloon. Shock, cardiogenic. Angioplasty.

\section{MÉTODOS}

\section{População do estudo}

Foram avaliados no presente estudo os pacientes submetidos a ICP em único centro (Instituto do Coração do Hospital das Clínicas da Faculdade de Medicina da Universidade de São Paulo - InCor/HCFMUSP, São Paulo, SP), no período de julho de 2001 a abril de 2009. Foram incluídos no estudo os pacientes submetidos a ICP de alto risco (tronco da coronária esquerda, disfunção ventricular grave - fração de ejeção < 30\% -, artérias coronárias derradeiras) e na vigência de choque cardiogênico que necessitaram suporte hemodinâmico com BIA. Esses pacientes foram acompanhados prospectivamente durante a evolução hospitalar.

\section{Procedimento}

Com relação à técnica de angioplastia, a antiagregação plaquetária utilizada foi clopidogrel em dose de ataque de 300 mg, seguido de 75 mg/dia por pelo menos 30 dias para pacientes tratados com stent convencional ou pelo menos por um ano nos pacientes tratados com stent farmacológico. Além disso, os pacientes receberam a prescrição para utilizar aspirina (100-300 mg/dia) indefinidamente. Após obtenção do acesso femoral em todos os casos $(\geq 6 \mathrm{~F}$ ) e introdução do cateter, foi administrada heparina nas doses preconizadas para se obter tempo de coagulação ativada $>250$ segundos (ou > 200 segundos se houvesse utilização de inibidores da glicoproteína IIb/IIla). Foi selecionado um stent de tamanho adequado para tratar as lesões. Stents de 2,5-4 mm de diâmetro e de 8-33 mm de comprimento estavam disponíveis para uso. A utilização de pré-dilatação com balão ou de stent direto ficou a critério do operador.

Foram utilizados os seguintes dispositivos de suporte circulatório: Maquet Lincor, Datascope System 97 e CS100 (Maquet, Rastatt, Alemanha). O BIA foi implantado pela técnica de Seldinger na artéria femoral, sendo realizada fluoroscopia rotineiramente para identificar o melhor local de punção. A manutenção de anticoagulação para a permanência do BIA ficou a critério do médico assistente responsável pelo paciente, entretanto foi recomendada a manutenção de anticoagulação, exceto se houvesse contraindicação, com heparina não-fracionada ou heparina de baixo peso molecular. A retirada ou manutenção do BIA foi 
indicada a critério do médico assistente, sendo utilizados parâmetros clínicos, laboratoriais e de monitorização invasiva com cateter de Swan-Ganz, o qual, contudo, não foi utilizado de rotina para guiar a terapêutica.

\section{Coleta e análise de dados}

Os dados da evolução hospitalar foram coletados durante a internação índice, seguindo o preenchimento de formulários previamente padronizados. A coleta incluiu características clínicas, resultados de exames laboratoriais, dados do procedimento invasivo (desde tempo do exame até as características angiográficas e da intervenção) e evolução clínica até a alta hospitalar. Todas as angiografias foram analisadas pelo Serviço de Hemodinâmica e Cardiologia Intervencionista do InCor/HCFMUSP, avaliando as angiografias inicial e de seguimento para determinar o sucesso do procedimento, medidas e características do vaso, e eventos adversos angiográficos. A aquisição de imagens foi realizada usando duas ou mais projeções angiográficas da estenose após a administração de nitrato. Essas projeções foram repetidas no momento da angiografia de seguimento nos casos em que houve necessidade. As características morfológicas qualitativas foram avaliadas utilizando critérios padronizados.

\section{Definições}

O choque cardiogênico foi definido por critérios clínicos, conforme o estudo Should We Emergently Revascularize Occluded Coronaries for Cardiogenic Shock (SHOCK), pela presença de hipotensão (pressão arterial sistólica $<90 \mathrm{mmHg}$ por pelo menos $30 \mathrm{mi}-$ nutos ou necessidade de medidas de suporte para manter pressão arterial sistólica $>90 \mathrm{mmHg}$ ) e hipoperfusão orgânica (débito urinário $<30 \mathrm{ml} /$ hora e frequência cardíaca > 60 bpm). ${ }^{14}$

O diagnóstico de IAM com supradesnível do segmento ST foi realizado na ocorrência de elevação persistente de ST > $1 \mathrm{~mm}$ em duas derivações contíguas ou bloqueio de ramo esquerdo novo ao eletrocardiograma. Angina estável foi considerada quando havia dor desencadeada por esforço, isquemia silenciosa documentada por prova funcional ou equivalente anginoso. Angina instável foi definida como dor em repouso, de início recente ou progressiva, sem os critérios eletrocardiográficos citados anteriormente; quando associada a elevação de marcadores de necrose miocárdica (creatina quinase fração MB - CK-MB ou troponina I), foi considerada como IAM sem supradesnivelamento do segmento ST. O sucesso angiográfico foi definido por redução da lesão-alvo < 30\%, com manutenção ou restabelecimento do fluxo anterógrado normal (TIMI 3). ${ }^{15}$

A morfologia das lesões foi classificada de acordo com as definições do American College of Cardiology/
American Heart Association (ACC/AHA), modificadas por Ellis et al. ${ }^{16}$. Foi considerada insuficiência renal aguda elevação de $25 \%$ da creatinina sérica basal ou aumento absoluto de $0,5 \mathrm{mg} / \mathrm{dl}$ de creatinina sérica entre 2 e 7 dias da realização do procedimento. ${ }^{17}$

As complicações vasculares foram relatadas como o desfecho composto por pseudoaneurisma, fístula arteriovenosa, hematoma importante no local do acesso (> $10 \mathrm{~cm}$ ), embolização distal e/ou isquemia relacionada ao local de punção, sangramento pelo acesso com queda de hemoglobina $\geq 2 \mathrm{mg} / \mathrm{dl}$ ou que requereu transfusão. $\mathrm{O}$ óbito foi definido como morte por qualquer causa.

\section{Análise estatística}

As variáveis contínuas foram descritas como média \pm desvio padrão, sendo comparadas com o teste de Wilcoxon. As variáveis categóricas foram representadas como sua porcentagem, sendo comparadas com o teste qui-quadrado. As características clínicas e angiográficas apresentadas nas Tabelas 1 e 2 foram incluídas em um modelo de regressão para detecção multivariada dos preditores de mortalidade.

Todos os testes foram bicaudais e um valor de $\mathrm{P}<0,05$ foi considerado significativo. As análises foram realizadas utilizando o programa SPSS 17.0 (SPSS Inc., Chicago, Estados Unidos).

\section{RESULTADOS}

No período de julho de 2001 a abril de 2009, foram incluídos consecutivamente 134 pacientes submetidos a passagem do BIA durante ICP de alto risco ou na vigência de choque cardiogênico no InCor/ HCFMUSP. Conforme demonstrado na Tabela 1, a média de idade foi de $64,7 \pm 12,5$ anos, com predomínio do sexo masculino $(67,9 \%)$. Além disso, 33,5\% da população era diabética, com antecedente de insuficiência cardíaca em $32,8 \%$ dos casos e fração de ejeção do ventrículo esquerdo reduzida $(38,6 \pm 16,1 \%)$. Quanto à terapia farmacológica adjunta, o uso de inibidor da glicoproteína IIb/IIla foi de 32,5\%, de clopidogrel antes do procedimento foi de $67,4 \%$, e de heparina, heparina não-fracionada ou heparina de baixo peso molecular, de $79,2 \%$. A maioria dos pacientes incluídos no estudo foi submetida à passagem de BIA no contexto de síndrome coronária aguda (IAM e angina instável em 84,3\% dos casos).

As características angiográficas e do procedimento são apresentadas na Tabela 2. Foi evidenciado que $68,6 \%$ dos pacientes apresentavam padrão de acometimento triarterial, sendo as lesões culpadas predominantemente do tipo B2 e C (90\%). O número de lesões tratadas por paciente foi de 1,6 $\pm 0,6$, com fluxo TIMI 2 ou 3 ao final do procedimento em $86,4 \%$ dos casos. O tempo médio de uso do BIA foi de 3,7 dias. 
TABELA 1

Características clínicas

\begin{tabular}{lc}
\hline & 134 pacientes \\
\hline Média de idade, anos & $64,7 \pm 12,5$ \\
Sexo masculino, \% & 67,9 \\
Diabetes, \% & 33,5 \\
Hipertensão arterial sistêmica, \% & 88,8 \\
Tabagismo atual, \% & 23,8 \\
Dislipidemia, \% & 70,8 \\
História familiar de doença & 33,5 \\
arterial coronária, \% & \\
Cirurgia de revascularização & 31,3 \\
miocárdica prévia, \% & \\
Intervenção coronária & 29,9 \\
percutânea prévia, \% & \\
Infarto agudo do miocárdio prévio, \% & 49,3 \\
Insuficiência renal crônica dialítica, \% & 3 \\
Acidente vascular cerebral prévio, \% & 6,7 \\
Insuficiência cardíaca congestiva, \% & 32,8 \\
Doença pulmonar obstrutiva crônica, \% & 3,7 \\
Hipotireoidismo, \% & 2,2 \\
Quadro clínico, \% & \\
Infarto agudo do miocárdio & 73,1 \\
Angina instável & 11,2 \\
Angina estável & 14,2 \\
FEVE, \% & $38,6 \pm 16,1$ \\
Farmacologia adjunta, \% & \\
Heparina & \\
Clopidogrel & \\
Inibidor da glicoproteína Ilb/llla & \\
\hline FEVE = fração de ejeção do ventrículo esquerdo. \\
\hline
\end{tabular}

Com respeito aos desfechos clínicos avaliados (Tabela 3$)$, ocorreram $18(13,4 \%)$ reinfartos, dos quais $9(6,7 \%)$ por trombose definitiva do stent, sendo $2(1,4 \%)$ pacientes encaminhados para cirurgia de revascularização miocárdica e 16 (11,9\%), para nova ICP. Dez $(55,5 \%)$ pacientes que tiveram reinfarto faleceram, e a mortalidade global intra-hospitalar foi de $61,2 \%$. A taxa de eventos cardíacos adversos maiores (ECAM) composta por óbito, reinfarto e revascularização de urgência foi de $67,2 \%$. Acidente vascular cerebral ocorreu em $5(3,7 \%)$ pacientes, 4 de etiologia isquêmica e 1 de etiologia hemorrágica.

Ocorreram ainda $17,2 \%$ de complicações vasculares, das quais $11,9 \%$ relacionadas a sangramento do acesso vascular e 4,5\% a alteração de perfusão periférica, com sinais de isquemia dos membros. Houve apenas um caso de pseudoaneurisma, corrigido cirurgicamente com sucesso. Insuficiência renal aguda ocorreu em $42,5 \%$ dos pacientes, dos quais $11,9 \%$ necessitaram diálise.
TABELA 2

Características angiográficas e do procedimento

134 pacientes

\begin{tabular}{lc}
\hline Padrão coronário, \% & \\
Uniarterial & 12 \\
Biarterial & 19,4 \\
Triarterial & 68,6 \\
Complexidade da lesão (ACC/AHA), \% & \\
A & 2,4 \\
B1 & 7,7 \\
B2 & 51,9 \\
C & 38 \\
Tipo de lesão, \% & \\
De novo & 97,8 \\
Reestenose & 2,2 \\
Lesões tratadas (lesões/paciente) & $1,6 \pm 0,6$ \\
Stents utilizados (stents/paciente) & $1,4 \pm 0,9$ \\
Diâmetro médio do stent, mm & $3,17 \pm 0,7$ \\
Comprimento médio do stent, mm & $26,7 \pm 18,3$ \\
Fluxo TIMI 2 ou 3 ao final & 86,4 \\
do procedimento, \% & \\
Tempo médio de permanência & 3,7 \\
do balão intra-aórtico, dias & \\
\hline
\end{tabular}

$\mathrm{ACC} / \mathrm{AHA}=$ American College of Cardiology/American Heart Association.

TABELA 3

Desfechos e complicações hospitalares

\begin{tabular}{lc}
\hline & 134 pacientes \\
\hline ECAM, \% & 67,2 \\
Óbito & 61,2 \\
Reinfarto & 13,4 \\
Revascularização de urgência & 13,4 \\
Acidente vascular cerebral isquêmico, \% & 3 \\
Acidente vascular cerebral hemorrágico, \% & 0,8 \\
Complicações vasculares, \% & 17,2 \\
Sangramento da via de acesso & 11,9 \\
Pseudoaneurisma ou & 0,8 \\
fístula arteriovenosa & 4,5 \\
Alteração de perfusão periférica & 7,5 \\
Necessidade de transfusão, \% & 42,5 \\
Insuficiência renal aguda, \% & 11,9 \\
Necessidade de diálise, \% & \\
\hline ECAM = eventos cardíacos adversos maiores. & \\
\hline
\end{tabular}

Por análise multivariada, conforme demonstrado na Tabela 4, são apresentadas as variáveis que interferiram independentemente na mortalidade hospita- 
TABELA 4

Variáveis que interferiram independentemente na mortalidade hospitalar

\begin{tabular}{|c|c|c|}
\hline Variável & $\begin{array}{l}\text { Razão de risco } \\
\text { (IC 95\%) }\end{array}$ & $\mathbf{P}$ \\
\hline \multicolumn{3}{|l|}{ Preditores de mortalidade } \\
\hline Idade $>65$ anos & $2,27(1,11-4,7)$ & 0,03 \\
\hline Intervenção coronária percutânea prévia & $2,41(1,05-5,5)$ & 0,05 \\
\hline Necessidade de diálise & $4,9(1,08-22,8)$ & 0,03 \\
\hline Padrão triarterial & $3,2(1,5-6,9)$ & 0,004 \\
\hline \multicolumn{3}{|l|}{ Preditores de sobrevida } \\
\hline FEVE normal & $0,97(0,94-0,99)$ & 0,03 \\
\hline Fluxo TIMI 2/3 ao final do procedimento & $0,3(0,1-0,91)$ & 0,041 \\
\hline Tempo de permanência do balão intra-aórtico > 3 dias & $0,3(0,13-0,71)$ & 0,007 \\
\hline
\end{tabular}

FEVE = fração de ejeção do ventrículo esquerdo.

lar. Observou-se que os preditores de mortalidade hospitalar foram: idade > 65 anos [odds ratio (OR) 2,27, intervalo de confiança de 95\% (IC 95\%) 1,11-4,7; $P=0,03)$, ICP prévia (OR 2,41, IC 95\% 1,05-5,5; $P=0,05)$, necessidade de diálise durante a internação (OR 4,9, IC 95\% 1,08-22,8; P = 0,03) e padrão coronário triarterial (OR 3,2, IC 95\% 1,5-6,9; $\mathrm{P}=0,004)$. Os preditores de sobrevida foram fração de ejeção do ventrículo esquerdo normal (OR 0,97, IC 95\% 0,94-0,99; $\mathrm{P}=0,03)$, fluxo TIMI $2 / 3$ ao final do procedimento (OR 0,3, IC 95\% 0,1-0,91; P=0,041), e tempo de permanência do BIA > 72 horas (OR 0,3, IC 95\% $0,13-0,71 ; P=0,007)$.

\section{DISCUSSÃO}

Na última década foi observada redução progressiva da mortalidade nas ICPs de alto risco, incluindo choque cardiogênico de etiologia isquêmica, ocasionada principalmente pelos avanços da terapia de reperfusão coronária percutânea e pela terapia farmacológica adjunta. ${ }^{18}$ Além disso, o uso do BIA nesse contexto, como terapia de suporte nas ICPs, passou a ser amplamente difundido e recomendado pelas diversas diretrizes atuais. ${ }^{10-12}$ Entretanto, esses dispositivos de assistência circulatória ainda têm sido pouco utilizados na prática clínica mundial, tanto pela escassez de benefício clínico em estudos randomizados como pela disponibilidade restrita aos grandes centros de cardiologia intervencionista.

Nesse sentido, procuramos avaliar, em um centro terciário de referência cardiovascular, o uso rotineiro do BIA como suporte adjunto durante as ICPs de alto risco, incluindo pacientes em vigência de choque cardiogênico de etiologia isquêmica. Verificou-se, nessa casuística, elevada mortalidade hospitalar $(61,2 \%)$ dos pacientes que necessitaram de BIA, além de alta taxa de complicações vasculares e necessidade de transfusão sanguínea. Além disso, foram identificadas di- versas variáveis que interferiram independentemente na mortalidade hospitalar.

Esses resultados podem ser explicados pelo elevado perfil de risco clínico dos pacientes estudados. Isso porque, em sua maioria, eram idosos, diabéticos, com função ventricular diminuída e com alta prevalência de revascularização coronária prévia (percutânea ou cirúrgica). Além disso, a maioria da população apresentava-se com síndrome coronária aguda (84,3\%), com predomínio de IAM (73,1\%). Com respeito ao padrão angiográfico, os pacientes em sua maioria eram triarteriais e com lesões complexas (tipos B2 e C).

Apesar de alta, a mortalidade verificada neste estudo é muito próxima daquela observada na literatura, descrita entre $42 \%$ e $65 \%$ dos pacientes, especialmente quando o BIA foi indicado durante ICP com estado de choque cardiogênico já estabelecido. ${ }^{19-21}$ Em subanálise do registro americano do NRMI- $2,{ }^{22}$ a mortalidade do IAM complicado por choque cardiogênico, mesmo em hospitais com ampla utilização do BIA, foi de 50,6\% vs. $65,4 \%$ em relação àqueles hospitais com menores taxas da utilização desse dispositivo de assistência circulatória $(P<0,001)$.

A estabilização hemodinâmica inicial, decorrente do aumento da perfusão coronária e consequente diminuição da área isquêmica, é o racional para o implante eletivo de BIA antes das ICPs de alto risco ou da evolução para choque cardiogênico. A utilização tardia desse suporte circulatório poderia atenuar seus potenciais efeitos benéficos, antes da obtenção da patência do vaso. No entanto, o melhor momento para implante desse dispositivo ainda é controverso na literatura. Estudos observacionais evidenciaram diminuição das complicações intraprocedimento e redução de eventos cardiovasculares e cerebrovasculares. ${ }^{19,23,24}$ Outro estudo, avaliando a inserção profilática do BIA, demonstrou que isso pode ser um preditor independente de 
sobrevida aos 6 meses. ${ }^{25}$ Entretanto, outros autores não evidenciaram redução dos desfechos compostos de morte, IAM, evento cerebrovascular ou necessidade de revascularização com a utilização profilática do BIA de rotina pré-intervenção percutânea de alto risco, ${ }^{26}$ como demonstrado recentemente pelo estudo randomizado Counterpulsation to Reduce Infarct Size Pre-PCI Acute Myocardial Infarction (CRISP AMI) ${ }^{27}$, em que não houve benefício em relação à redução da área isquêmica com o uso profilático do BIA em pacientes com infarto anterior extenso sem choque cardiogênico.

Neste estudo, apenas nos casos de ICP de alto risco os pacientes receberam o BIA profilaticamente, o que representou a minoria da população. Mesmo assim, por causa dessa controvérsia na literatura, não se sabe se o uso "profilático" de BIA poderia ter modificado a alta taxa de mortalidade da população estudada.

A contrapulsação com o BIA é um procedimento invasivo, que pode produzir complicações, especialmente vasculares e hemorrágicas, no local do acesso. ${ }^{28}$ Apesar disso, a maior experiência dos operadores aliada à evolução da técnica e ao desenvolvimento tecnológico, com melhor perfil dos materiais, têm reduzido a incidência de complicações, especialmente a isquemia dos membros. ${ }^{29}$ As taxas de complicação, porém, ainda são elevadas, podendo ser preditas pelo tempo de permanência do BIA, IAM, idade > 65 anos e fração de ejeção do ventrículo esquerdo $<0,30$, fatores bastante prevalentes nesta população. ${ }^{30}$ Além disso, como já descrito na literatura atual, a cada 10 anos de incremento na idade do paciente há aumento de $49 \%$ a $60 \%$ na mortalidade. ${ }^{31,32}$

Nesta casuística também puderam ser identificadas algumas variáveis que interferiram independentemente na mortalidade hospitalar. Nesse sentido, verificou-se, como preditores de mortalidade hospitalar, idade $>65$ anos, ICP prévia, necessidade de diálise durante internação e padrão coronário triarterial. Por outro lado, foram fatores protetores fração de ejeção do ventrículo esquerdo normal, fluxo TIMI 2/3 ao final do procedimento, e tempo de permanência do BIA > 72 horas. Ressalta-se que o tempo de anticoagulação não esteve associado aos desfechos clínicos analisados. Essas informações são fundamentais para que se possa estratificar mais adequadamente os pacientes com relação ao risco cardiovascular.

A questão sobre o uso de BIA por mais de 72 horas ter sido preditor independente de sobrevida é um dado interessante. Se por um lado sabe-se que as primeiras 24 horas do choque cardiogênico são relacionadas a maior mortalidade, ${ }^{33}$ nesta amostra apenas $26,8 \%$ dos óbitos ocorreram em 24 horas e 41,5\% em até 48 horas. No entanto, não dispomos dos dados hemodinâmicos e clínicos individualizados que motivaram a retirada do BIA de cada paciente; nesse sentido, o uso prolongado do BIA poderia ser um marcador de pacientes menos graves, que sobreviveram à fase crítica inicial. Diante dessas considerações, mesmo o uso $>72$ horas tendo sido preditor independente de sobrevida, não se pode recomendar seu uso mais prolongado, até porque isso também é sabidamente relacionado ao aumento das taxas de complicação vascular. ${ }^{34}$

Portanto, os altos índices de mortalidade e de complicações vasculares e hemorrágicas devem-se ao elevado perfil de risco dos pacientes, à gravidade de apresentação e à necessidade de potente farmacologia antiplaquetária adjunta, e não ao implante de BIA, que permanece indicado como importante dispositivo de assistência nesse grupo de pacientes. Independentemente do tipo de recomendação e orientação, o uso de BIA tornou-se uma tecnologia bem estabelecida mais de quatro décadas após sua introdução. Na era da medicina baseada em evidências, ainda há espaço para o julgamento clínico, no sentido de antever os pacientes e as condições em que seu uso "profilático" pode resultar em benefícios clínicos, tendo em vista a ainda alta taxa de mortalidade dos pacientes que necessitam de sua utilização. Talvez a utilização de novos dispositivos de assistência circulatória possam, no futuro, reduzir as taxas de mortalidade desses pacientes. ${ }^{35}$

\section{Limitações do estudo}

Dentre as limitações do presente estudo destacam-se sua característica observacional, sem grupo controle. Contudo, por se tratar de população específica e altamente selecionada, considerou-se a casuística significativa para o uso desse dispositivo de assistência circulatória na realização de ICP de alto risco.

Apesar de a angiografia de rotina pré-inserção do BIA ser importante para avaliação de doença vascular periférica e identificação do membro com melhor anatomia para o implante do BIA, esse procedimento não foi realizado de rotina, pois tratava-se de um grupo de pacientes de alto risco, com instabilidade hemodinâmica, cuja importância em minimizar volume de contraste e a rapidez para estabilização clínica se sobrepuseram à avaliação rotineira de insuficiência arterial periférica.

\section{CONCLUSÕES}

O uso de BIA em pacientes submetidos a ICP de alto risco esteve associado a elevada mortalidade e alto número de complicações vasculares e sangramentos. Fatores clínicos, angiográficos e do procedimento podem identificar variáveis que interferem independentemente na mortalidade hospitalar.

\section{CONFLITO DE INTERESSES}

Os autores declaram não haver conflito de interesses relacionado a este manuscrito. 


\section{REFERÊNCIAS}

1. Mansur Ade P, Favarato D, Avakian SD, Ramires JA. Trends in ischemic heart disease and stroke death ratios in Brazilian women and men. Clinics (Sao Paulo). 2010;66(11):1143-7.

2. Brasil. Ministério da Saúde. DATASUS. Informações de Saúde. Mortalidade [Internet]. Brasília; 2007 [citado 2011 ago. 15]. Disponível em: http://tabnet.datasus.gov.br/cgi/tabcgi.exe?sim/ cnv/obtbr.def

3. Matte BS, Bergoli LCC, Balvedi JA, Zago AC. Perfil da intervenção coronária percutânea no infarto agudo do miocárdio com supradesnivelamento do segmento ST no Brasil de 2006 a 2010 - Registro CENIC. Rev Bras Cardiol Invasiva. 2011; 19(2):131-7.

4. Hochman JS, Boland J, Sleeper LA, Porway M, Brinker J, Col $\mathrm{J}$, et al. Current spectrum of cardiogenic shock and effect of early revascularization on mortality. Results of an International Registry. SHOCK Registry Investigators. Circulation. 1995;91(3): 873-81.

5. Hollenberg SM, Kavinsky CJ, Parrillo JE. Cardiogenic shock. Ann Intern Med. 1999;131(1):47-59.

6. Alexander JH, Reynolds HR, Stebbins AL, Dzavik V, Harrington RA, Van de Werf $F$, et al. Effect of tilarginine acetate in patients with acute myocardial infarction and cardiogenic shock: the TRIUMPH randomized controlled trial. JAMA. 2007; 297(15):1657-66.

7. Babaev A, Frederick PD, Pasta DJ, Every N, Sichrovsky T, Hochman JS; NRMI Investigators. Trends in management and outcomes of patients with acute myocardial infarction complicated by cardiogenic shock. JAMA. 2005;294(4):448-54.

8. Valim LR, Lopes ACA, Bienert IRC, Ribeiro HB, Campos CA, Esper RB, et al. Infarto agudo do miocárdio complicado por choque cardiogênico: efeito da circulação colateral nos resultados da intervenção coronária percutânea primária Dados do Registro InCor. Rev Bras Cardiol Invasiva. 2011;19(2): 166-71.

9. Moulopoulos SD, Topaz S, Kolff WJ. Diastolic balloon pumping (with carbon dioxide) in the aorta-a mechanical assistance to the failing circulation. Am Heart J. 1962;63:669-75.

10. Mattos LA, Lemos Neto PA, Rassi A Jr, Marin-Neto JA, Sousa AGMR, Devito FS, et al. Diretrizes da Sociedade Brasileira de Cardiologia - Intervenção Coronária Percutânea e Métodos Adjuntos Diagnósticos em Cardiologia Intervencionista (II Edição - 2008). Arq Bras Cardiol. 2008;91(4 Supl 1):1-58.

11. Silber S, Albertsson P, Aviles FF, Comici PG, Colombo A, Hamm C, et al. Guidelines for percutaneous coronary interventions. The Task Force for Percutaneous Coronary Interventions of the European Society of Cardiology. Eur Heart J. 2005; 26(8):804-47.

12. Smith SC Jr, Feldman TE, Hirshfeld JW Jr, Jacobs AK, Kern MJ, King SB $3^{\text {rd }}$, et al. ACC/AHA/SCAI 2005 guideline update for percutaneous coronary intervention: a report of the American College of Cardiology/American Heart Association Task Force on Practice Guidelines (ACC/AHA/SCAI Writing Committee to Update 2001 Guidelines for Percutaneous Coronary Intervention). Circulation. 2006;113(7):e166-286.

13. Goldberg RJ, Samad NA, Yarzebski J, Gurwitz J, Bigelow C, Gore JM. Temporal trends in cardiogenic shock complicating acute myocardial infarction. N Engl J Med. 1999;340(15): 1162-8.

14. Hochman JS, Sleeper LA, Webb JG, Sanborn TA, White HD, Talley JD, et al. Early revascularization in acute myocardial infarction complicated by cardiogenic shock. SHOCK Investigators. Should We Emergently Revascularize Occluded Coronaries for Cardiogenic Shock. N Engl J Med. 1999;341(9):625-34.

15. TIMI Study Group. The Thrombolysis in Myocardial Infarction
(TIMI) trial. Phase I findings. N Engl J Med. 1985;312(14): 932-6.

16. Ellis SG, Vandormael MG, Cowley MJ, DiSciascio G, Deligonul U, Topol EJ, et al. Coronary morphologic and clinical determinants of procedural outcome with angioplasty for multivessel coronary disease. Implications for patient selection. Multivessel Angioplasty Prognosis Study Group. Circulation. 1990;82(4):1193-202.

17. Lameire N, Van Biesen W, Vanholder R. Acute renal failure. Lancet. 2005;365(9457):417-30.

18. Jeger RV, Radovanovic D, Hunziker PR, Pfisterer ME, Stauffer JC, Erne P, et al. Ten-year trends in the incidence and treatment of cardiogenic shock. Ann Intern Med. 2008;149(9):618-26.

19. Abdel-Wahab M, Saad M, Kynast J, Geist V, Sherif MA Richardt G, et al. Comparison of hospital mortality with intra-aortic balloon counterpulsation insertion before versus after primary percutaneous coronary intervention for cardiogenic shock complicating acute myocardial infarction. Am J Cardiol. 2010;105(7):967-71.

20. Barron HV, Every NR, Parsons LS, Angeja B, Goldberg RJ, Gore JM, et al. The use of intra-aortic balloon counterpulsation in patients with cardiogenic shock complicating acute myocardial infarction: data from the National Registry of Myocardial Infarction 2. Am Heart J. 2001;141(6):933-9.

21. Cheng JM, Valk SD, den Uil CA, van der Ent M, Lagrand WK, van de Sande $M$, et al. Usefulness of intra-aortic balloon pump counterpulsation in patients with cardiogenic shock from acute myocardial infarction. Am J Cardiol. 2009;104(3): $327-32$.

22. Chen EW, Canto JG, Parsons LS, Peterson ED, Littrell KA Every NR, et al. Relation between hospital intra-aortic balloon counterpulsation volume and mortality in acute myocardial infarction complicated by cardiogenic shock. Circulation. 2003; 108(8):951-7.

23. Briguori C, Sarais C, Pagnotta P, Airoldi F, Liistro F, Sgura $\mathrm{F}$, et al. Elective versus provisional intra-aortic balloon pumping in high-risk percutaneous transluminal coronary angioplasty. Am Heart J. 2003;145(4):700-7.

24. Brodie BR, Stuckey TD, Hansen C, Muncy D. Intra-aortic balloon counterpulsation before primary percutaneous transluminal coronary angioplasty reduces catheterization laboratory events in high-risk patients with acute myocardial infarction. Am J Cardiol. 1999;84(1):18-23.

25. Mishra S, Chu WW, Torguson R, Wolfram R, Deible R, Suddath WO, et al. Role of prophylactic intra-aortic balloon pump in high-risk patients undergoing percutaneous coronary intervention. Am J Cardiol. 2006;98(5):608-12.

26. Perera D, Stables R, Thomas M, Booth J, Pitt M, Blackman $D$, et al. Elective intra-aortic balloon counterpulsation during high-risk percutaneous coronary intervention: a randomized controlled trial. JAMA. 2010;304(8):867-74.

27. Patel MR, Smalling RW, Thiele $H$, Barnhart HX, Zhou $Y$, Chandra $\mathrm{P}$, et al. Intra-aortic balloon counterpulsation and infarct size in patients with acute anterior myocardial infarction without shock: The CRISP AMI Randomized Trial. JAMA. 2011;306(12):1329-37.

28. Erdogan HB, Goksedef D, Erentug V, Polat A, Bozbuga N, Mansuroglu D, et al. In which patients should sheathless IABP be used? An analysis of vascular complications in 1211 cases. J Card Surg. 2006;21(4):342-6

29. Cohen M, Ferguson JJ 3rd, Freedman RJ Jr, Miller MF, Reddy RC, Ohman EM, et al. Comparison of outcomes after 8 vs. 9.5 French size intra-aortic balloon counterpulsation catheters based on 9,332 patients in the prospective Benchmark registry. Catheter Cardiovasc Interv. 2002;56(2):200-6.

30. Christenson JT, Cohen M, Ferguson JJ $3^{\text {rd }}$, Freedman RJ, Miller 
MF, Ohman EM, et al. Trends in intraaortic balloon counterpulsation complications and outcomes in cardiac surgery. Ann Thorac Surg. 2002;74(4):1086-90; discussion 90-1.

31. Berger PB, Tuttle RH, Holmes DR Jr, Topol EJ, Aylward PE, Horgan $\mathrm{JH}$, et al. One-year survival among patients with acute myocardial infarction complicated by cardiogenic shock, and its relation to early revascularization: results from the GUSTO-I trial. Circulation. 1999;99(7):873-8.

32. Hasdai D, Holmes DR Jr, Califf RM, Thompson TD, Hochman JS, Pfisterer $M$, et al. Cardiogenic shock complicating acute myocardial infarction: predictors of death. GUSTO Investigators. Global Utilization of Streptokinase and Tissue-Plasminogen Activator for Occluded Coronary Arteries. Am Heart J. 1999; 138(1 Pt 1):21-31.
33. Webb JG, Sleeper LA, Buller CE, Boland J, Palazzo A, Buller $E$, et al. Implications of the timing of onset of cardiogenic shock after acute myocardial infarction: a report from the SHOCK Trial Registry. SHould we emergently revascularize Occluded Coronaries for cardiogenic shocK? J Am Coll Cardiol. 2000;36(3 Suppl A):1084-90.

34. Christenson JT, Sierra J, Romand JA, Licker M, Kalangos A. Long intraaortic balloon treatment time leads to more vascular complications. Asian Cardiovasc Thorac Ann. 2007;15(5): 408-12.

35. Sjauw KD, Konorza T, Erbel R, Danna PL, Viecca M, Minden $\mathrm{HH}$, et al. Supported high-risk percutaneous coronary intervention with the Impella 2.5 device the Europella registry. J Am Coll Cardiol. 2009;54(25):2430-4. 